\title{
How Can We Predict pT3a Kidney Cancer and What Does It Mean?
}

Commentary for the article "Radiological Correlates of pT3a Kidney Cancer: Importance of Irregular Tumor Sinus Border"

\section{Nirmish Singla, MD, MSc}

Departments of Urology and Oncology, Johns Hopkins University Baltimore, MD

https://doi.org/10.52733/KCJ19n4-c

$\mathrm{T}$ The ability to predict pathologically advanced renal cell carcinoma (RCC) within the primary tumor upfront can be helpful to guide prognostic counseling and hold implications for both surgical approach and multimodal therapeutic strategies. Herein, the investigators undertook a comprehensive assessment of radiographic features predictive of pT3a stage by querying 11 radiological findings across a robust retrospective cohort of patients with RCC. They found that an irregular tumor-sinus border (ITSB) correlated most strongly with $\mathrm{pT} 3 \mathrm{a}$ stage and recurrence-free survival (RFS).

While not explicitly investigated in the present study, predictors for pathological upstaging of small renal masses (cT1a) to pT3a at surgical resection have been investigated previously. This information has been used by some to help dictate the role for or against active surveillance and for or against a nephron-sparing surgical approach. It is important to note, however, that not all $\mathrm{pT}_{3} \mathrm{a} \mathrm{RCC}$ are created equal. First, focal microscopic local invasion constituting $\mathrm{pT} 3 \mathrm{a}$ acts different clinically compared to gross invasion constituting $\mathrm{pT} 3 \mathrm{a}$ tumors. Furthermore, the pT3a category is quite broad and includes (1) renal sinus fat invasion, (2) perirenal fat invasion, or (3) invasion of the renal vein or segmental branches. The location and pattern of local invasion warrants additional attention biologically to elucidate how similar these subcategories of $\mathrm{pT}_{3}$ a truly are and whether further sub-stratification of the pT3a stage is needed.

The cohort investigated notably consisted of either CT or MRI to determine radiographic correlates of pT3a. This naturally begs the question of whether one modal- ity is more reliable than the other in interpreting radiographic parameters such as ITSB. This is important to note, as future applications of this work would be presumably couched in machine learning and radiomic approaches to predicting $\mathrm{pT}_{3} \mathrm{a}$ stage.

Finally, the role for perioperative systemic therapy in reducing RFS among select, high-risk patients has been a topic of considerable contemporary interest. The adjuvant space has enjoyed recent FDA approval of the immune checkpoint inhibitor pembrolizumab based on the results of the KEYNOTE-564 trial. However, the ability to more accurately predict aggressive, locally advanced disease upfront may lend support to neoadjuvant strategies in select patients with RCC, and in this context we eagerly await the results of the PROSPER-RCC trial among others.

\footnotetext{
* Correspondence: Nirmish Singla, MD, MSc. Departments of Urology and Oncology,

The James Buchanan Brady Urological Institute, The Johns Hopkins University School of Medicine, 6oo North Wolfe Street, Park 213, Baltimore, MD 21287, Email: nsingla2@jhmi.edu. Disclosures: None
} 